\title{
OBTENÇÃO DO SALDO DE RADIAÇÃO EM ÁREAS DE PASTAGEM E FLORESTA NA AMAZÔNIA (ESTAÇÃO SECA) ATRAVÉS DO SENSOR MODIS
}

\author{
CARLOS ANTONIO COSTA DOS SANTOS ${ }^{1}$, RAIANNY L. NASCIMENTO WANDERLEY ${ }^{2}$, \\ ARGEMIRO LUCENA ARAÚJO ${ }^{1}$, BERGSON GUEDES BEZERRA ${ }^{3}$ \\ ${ }^{1}$ Universidade Federal de Campina Grande (UFCG), Campina Grande, PB, Brasil \\ ${ }^{2}$ Universidade de São Paulo, Instituto de Astronomia, Geofísica e Ciências Atmosféricas, São Paulo, SP, \\ Brasil \\ ${ }^{3}$ Universidade Federal do Rio Grande do Norte, Centro de Ciências Exatas, Natal, RN, Brasil
}

carlostorm@gmail.com, yakurin@gmail.com, argemirofera@gmail.com, bergson.bezerra@gmail.com

Recebido Fevereiro de 2013 - Aceito Novembro de 2013

\begin{abstract}
RESUMO
O principal objetivo deste estudo foi analisar de forma comparativa a estimativa do saldo de radiação à superfície $(\mathrm{Rn})$ a partir de imagens orbitais com dados de superfície medidos, bem como os componentes do Rn, para dois diferentes tipos de cobertura vegetal (pastagem e floresta) sob condições atmosféricas tropical-úmida. Este estudo foi realizado no estado de Rondônia, no noroeste do Brasil. Para realizar as análises através de técnicas de sensoriamento remoto foram utilizadas sete imagens orbitais do sensor MODIS a bordo do satélite Aqua, para a aplicação do algoritmo e os dados de superfície medidos por duas torres micrometeorológicas, sendo instaladas em área de pastagem e de floresta. Os principais resultados obtidos a partir das imagens MODIS mostram erro percentual (EP) máximos e mínimos coerentes para o Rn de 3\% e 0,02\%, para a área de floresta e para a área de pastagem apresentou valores de EP de $10 \%$ e $0,7 \%$. Esses resultados mostram que o sensoriamento remoto orbital é uma ferramenta importante e eficaz em estudos hidrológicos e ambientais.

Palavras-chave: sensoriamento remoto, micrometeorologia, SEBAL, balanço de radiação
\end{abstract}

\begin{abstract}
OBTAINING THE NET RADIATION IN PASTURES AND FOREST AREAS IN AMAZON (DRY SEASON) BY THE MODIS SENSOR

The main objective of this study was to analyze comparatively the estimate of net radiation at surface $(\mathrm{Rn})$ from orbital images with surface measured data as well as the components of Rn, for two different types of vegetation (pasture and forest) under tropical-humid climate. This study was conducted at Rondônia State in northwestern Brazil. To carry out the analysis using remote sensing techniques seven orbital images of MODIS sensor aboard the Aqua satellite were used for the algorithm implementation, and the measured surface data at two micrometeorological towers installed in the pasture and forest locations. The main results obtained from MODIS images show consistent maximum and minimum percentage error (PE) for Rn , 3\% and $0.02 \%$ for the forest area and $10 \%$ and $0.7 \%$ for the pasture area. These results show that remote sensing is an important and effective tool in environmental and hydrological studies.
\end{abstract}

Keywords: remote sensing, micrometeorology, SEBAL, net radiation 


\section{INTRODUÇÃO}

A quantificação do saldo de radiação à superfície (Rn) é essencial para o estudo dos processos de interação entre a superfície e a atmosfera da Terra. Estimar o Rn e seus componentes (radiação de ondas curtas e longas incidente, refletida e/ou emitida) tem implicação na hidrologia, monitoramento climático, previsão de tempo, meteorologia agrícola, energia renovável entre outros (Bisht et al., 2005; Bisht e Bras, 2011). O Rn é a diferença entre os fluxos radiativos (ondas longas e curtas) à superfície e controla os processos de evaporação, fotossíntese e aquecimento do solo e do ar. Além disso, o Rn é importante para o desenvolvimento da camada limite planetária (Roy e Avissar, 2002).

Dados de sensoriamento remoto obtidos por satélites de órbitas polares e geoestacionárias oferecem alta cobertura espacial e temporal da terra, atmosfera e oceanos. Bisht e Bras (2010) mostram que numerosos estudos têm estimado o Rn ou suas componentes usando dados de satélite. Entretanto, um relatório técnico recente do IPCC (Intergovernamental Panel for Climate Change) (IPCC, 2008) estudando os impactos das mudanças climáticas nos recursos hídricos mostrou a necessidade de informações que são cruciais para melhorar o entendimento e a modelagem das mudanças climáticas relacionadas ao ciclo hidrológico. Assim, o sensor MODIS (MODerate-resolution Imaging Spectroradiometer), a bordo dos satélites de órbita polar Aqua e Terra da NASA (National Aeronautics and Space Administration), provê cobertura global com aproximadamente quatro passagens por dia, oferecendo produtos relacionados à atmosfera, solo e oceano. Essas características tornam o MODIS de fundamental importância para estudos ambientais, principalmente, sobre regiões do globo em que não dispõem de informações suficientes para os referidos estudos.

Assim, o sensoriamento remoto constitui uma ferramenta inovadora capaz de observar quantitativamente os processos físico-químicos da superfície terrestre em grande escala espacial e apresentando um bom custo-benefício (Cai e Sharma, 2010), visto que muitos produtos radiométricos podem ser adquiridos gratuitamente. Portanto, vários estudos têm tentado estimar Rn através da combinação de sensoriamento remoto com dados de observações atmosféricas e da superfície (Rimóczi-Paal, 2005; Samani et al., 2007; Ryu et al., 2008; Wang e Liang, 2009).

Nessa visão, Bastiaanssen et al. (1998) estudaram e apresentaram as funcionalidades e aplicações do algoritmo SEBAL (Surface Energy Balance Algorithm for Land) para obter valores de Rn, com variações no espaço e no tempo. O SEBAL é um algoritmo que visa realizar estimativas dos componentes do balanço de energia, culminando com a integração do valor instantâneo (momento da passagem do satélite) do fluxo de calor latente para valor diário da evapotranspiração baseado em combinações de relações empíricas e parametrizações físicas (Bastiaanssen et al., 1998; Bastiaanssen, 2000; Allen et al., 2002). Os vários testes com o SEBAL em diferentes ecossistemas e em diferentes condições climáticas, durante as últimas décadas, mostram que a técnica tem sido cientificamente consistente. Ahmad et al. (2009) aplicaram esse algoritmo para realizar um diagnóstico do desempenho da irrigação e produtividade da água através de dados de satélites no Paquistão. Zhang et al. (2008) demonstraram a eficácia do algoritmo SEBAL após aplicá-lo no comparativo das estimativas dos fluxos de calor, através de imagens do sensores MODIS/Aqua (1 $\mathrm{km})$ e TM/Landsat-5 (30 m). No entanto, Mendonça et al. (2009) aplicaram o algoritmo SEBAL e imagens MODIS para estimar o fluxo de calor no solo (G) na região Norte Fluminense-RJ, Brasil e observaram que o algoritmo superestimou os valores medidos sobre as áreas ocupadas com cana-de-açúcar e subestimou os valores medidos nas áreas ocupadas com coqueiro anão verde. Ferreira e Meirelles (2011) na tentativa de implementar o SEBAL para estimativas da evapotranspiração na Mesorregião do Sul Goiano, observaram que o algoritmo requer a associação de dados detalhados de uso do solo para melhorias das referidas estimativas. Assim, são observadas as limitações do algoritmo em alguns estudos desenvolvidos no Brasil, carecendo assim, de mais investigações científicas.

Aguiar (2007) evidencia que diversos estudos já foram desenvolvidos para analisar o balanço de radiação em áreas de floresta e de pastagem na Região Amazônica. Entretanto, com o aumento considerável da área desmatada na Amazônia Legal, faz-se necessário a continuidade de estudos dessa natureza com o objetivo de avaliar as alterações nas características radiativas daquela região, devido ao desmatamento e suas consequências nos processos biológicos, hidrológicos e biogeoquímicos. Nesse contexto a utilização dos dados de sensoriamento remoto permitirá um melhor entendimento da distribuição espacial desses parâmetros. Assim, o objetivo deste estudo é estimar o saldo de radiação $(\mathrm{Rn})$ em áreas de floresta e pastagem no município de Ji-Paraná, estado de Rondônia utilizando o método do algoritmo SEBAL e produtos do satélite MODIS/Aqua, e comparar os resultados com valores medidos em superfície nas torres micrometeorológicas do Programa de Grande Escala da Biosfera-Atmosfera na Amazônia (LBA/INPA).

\section{MATERIAL E MÉTODOS}

\section{1 Área de estudo}

O presente estudo foi realizado no Estado de Rondônia, no noroeste do Brasil. A área de floresta, é chamada de Reserva Biológica do Jaru (Rebio-Jaru), localizada nas coordenadas 
$10^{\circ} 04^{\prime} 48.00$ " S e $61^{\circ} 55^{\prime} 48.00$ " W e $120 \mathrm{~m}$ acima do nível médio do mar. A área de pastagem está localizada na fazenda de criação de gado denominada Fazenda Nossa Senhora (FNS), apresentando coordenadas geográficas de $10^{\circ} 45^{\prime} 0.00^{\prime}$ ' S e $62^{\circ}$ 22' 12.00 " W e elevação de 293 m com relação ao nível médio do mar. Na Figura 1, encontram-se as localizações geográficas das torres micrometeorológicas instaladas dentro da área de estudo, em Rondônia, nos diferentes tipos de cobertura do solo (pastagem e floresta).

A média anual da umidade relativa do ar varia de $80 \%$ a $90 \%$ no verão, e em torno de $75 \%$, no outono - inverno. A evapotranspiração potencial (ETP) é alta durante todo o ano, apresentando valores superiores a $100 \mathrm{~mm} / \mathrm{mês}$. O total anual da ETP só atinge valores superiores aos da precipitação mensal nos meses de maio, junho, julho e agosto.

Os principais fenômenos atmosféricos ou mecanismo dinâmico que atuam no regime pluvial do Estado de Rondônia são: as Altas Convecções diurnas (água evaporada no local e a evapotranspiração resultante do aquecimento das superfícies das águas, florestas e vegetação), associadas aos fenômenos atmosféricos de larga escala: a Alta da Bolívia - AB (anticiclone que se forma nos altos níveis da atmosfera $(200 \mathrm{hPa})$ durante os meses de verão e situa-se sobre o altiplano boliviano), a Zona de Convergência Intertropical - ZCIT e as Linhas de Instabilidade - LIs (conglomerados de nuvens cumulonimbus que se formam na costa norte - nordeste do oceano Atlântico, devido a circulação da brisa marítima).

Nos meses de inverno a brisa pluvial da Bacia Amazônica (circulação local que ocorre nos baixos níveis da atmosfera) e os aglomerados convectivos de meso e grande escalas, associados com a penetração de sistemas frontais, advindo da região Sul e Sudeste do Brasil, são os principais mecanismos responsáveis pelas chuvas de baixa intensidade, enquanto que, nos meses de verão ocorre o período mais chuvoso, onde se observa uma grande atividade convectiva causada por uma maior incidência de radiação solar, durante o ano. O período chuvoso ocorre entre os meses de outubro a abril, e o período mais seco de junho a agosto. Maio e setembro são meses de transição. $\mathrm{O}$ clima predominante dessa região é o clima equatorial, o mais chuvoso do Brasil, com a maior parte do ano quente e úmido, e aproximadamente 3 meses de seca. As temperaturas médias anuais variam entre $24^{\circ}$ e $26^{\circ} \mathrm{C}$, podendo atingir temperaturas máximas de $33^{\circ} \mathrm{C}$ e mínimas de $10^{\circ} \mathrm{C}$. A precipitação pluvial anual varia de 1400 a $2500 \mathrm{~mm}$.

As torres que realizam as medições micrometeorológicas estão localizadas uma na região de floresta e a outra na região de pastagem. A lista das variáveis meteorológicas e da altura dos instrumentos é apresentada na Tabela 1.

\subsection{Material}

Para a obtenção do $\mathrm{Rn}$ através de imagens com baixa resolução espacial (250-500m no espectro solar e $1 \mathrm{~km}$ no espectro térmico) foram utilizados os produtos MYD07, MYD09_A1, MYD11_A2 do sensor MODIS a bordo do satélite Aqua (Tabela 2), correspondente ao ano de 2008, dos tiles h11v09 e h11v10 que foram utilizados para realizar mosaicos da região e com horário de passagem às 14:35h e 15:25h, horário local.

Os dados de superfície utilizados para a análise comparativa foram coletados nas torres localizadas nas duas áreas de estudo e são referentes ao ano de 2008 em intervalos de 10 minutos. Esses dados foram cedidos pelo LBA/INPA. O software utilizado para o processamento das imagens foi o

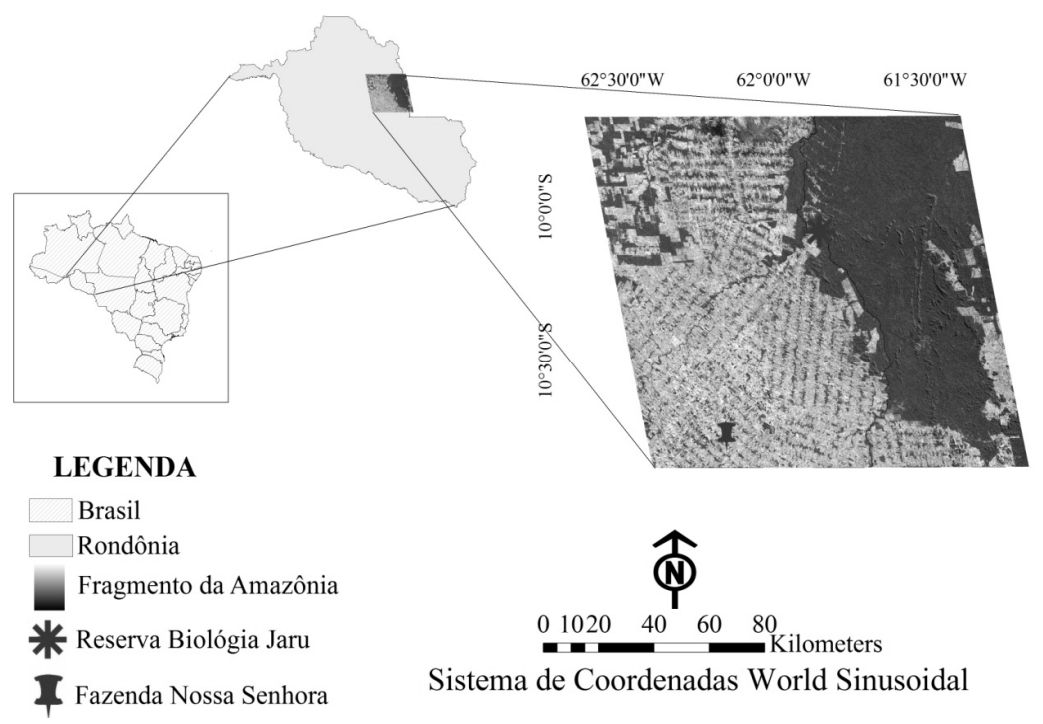

Figura 1 - Localização geográfica da área de estudo, em Rondônia, com destaque para diferentes coberturas de terra (pastagem e floresta). 
Tabela 1 -Descrição das variáveis meteorológicas e da altura dos instrumentos nas superfícies de pastagem e floresta em Rondônia

\begin{tabular}{llcc}
\hline \multicolumn{1}{c}{ Variáveis meteorológicas } & \multicolumn{1}{c}{ Instrumentos } & $\begin{array}{c}\text { Altura (m) } \\
\text { Pastagem (FNS) }\end{array}$ & $\begin{array}{c}\text { Altura (m) } \\
\text { Rebio-Jaru (Floresta) }\end{array}$ \\
\hline Radiação de onda curta incidente e refletida & Piranômetro modelo CM21 (Kipp \& Zonen) & 6.5 & 19.03 \\
Radiação de onda longa emitida e incidente & Piranômetro modelo CG1 (Kipp \& Zonen) & 6.5 & 60.0 \\
Temperatura e umidade do ar & Termohigrômetro modelo HMP35A (Vaisala) & 8.3 & 9.3 \\
Velocidade do vento & Anemômetro modelo A100R (Vector) & 8.0 & 61.1 \\
Temperatura da superfície radiativa & Sensor infravermelho modelo KT15 (Heimann) & 0.5 & 59.1 \\
Precipitação & Pluviômetro & & 60.3 \\
\hline
\end{tabular}

Fonte: Von Randow et al. (2004)

Tabela 2 - Descrição dos produtos MODIS/Aqua utilizados nesse estudo

\begin{tabular}{|c|c|c|c|c|c|}
\hline Produto & Descrição & $\begin{array}{c}\text { Fator } \\
\text { Multiplicativo }\end{array}$ & $\begin{array}{c}\text { Fator } \\
\text { adicional }\end{array}$ & $\begin{array}{c}\text { Resolução (espacial } \\
\text { e temporal) }\end{array}$ & Unidades \\
\hline \multirow{3}{*}{ MYD11A1 } & Temperatura da superfície & 0,02 & - & \multirow{3}{*}{$\begin{array}{c}1000 \mathrm{~m} \\
\text { diária }\end{array}$} & Kelvin \\
\hline & Emissividade das bandas 31 e 32 & 0,002 & 0,490 & & Adimensional \\
\hline & Hora da passagem do satélite & 0,1 & & & Hora \\
\hline \multirow[b]{2}{*}{ MYD09GA } & Refletância da superfície & 0,0001 & - & $\begin{array}{l}500 \mathrm{~m} \\
\text { diária }\end{array}$ & Adimensional \\
\hline & Ângulo zenital solar & 0,01 & - & $\begin{array}{c}1000 \mathrm{~m} \\
\text { diária }\end{array}$ & Grau \\
\hline \multirow{2}{*}{ MYD07_L2 } & Temperatura do ar & 0,01 & $1,5 \times 10^{4}$ & \multirow{2}{*}{$\begin{array}{l}5 \mathrm{~km} \\
\text { diária }\end{array}$} & Kelvin \\
\hline & Temperatura do ponto do orvalho & 0,01 & $1,5 \times 10^{4}$ & & Kelvin \\
\hline
\end{tabular}

Fonte: http://modis.gsfc.nasa.gov/

ERDAS Imagine e para a confecção dos mapas temáticos foi utilizado o SIG ArcGis.

Para realizar o processamento para a obtenção do Rn, foram utilizados dados gerados pelo satélite MODIS/Aqua para todo o ano de 2008, o que resultou em 45 imagens. Dentre estas, foram selecionados apenas os que não apresentavam nuvens sobre os pontos de coordenadas conhecidas, referentes às torres micrometeorológicas. Essa seleção resultou em 7 imagens, referentes aos dias de ordem do ano (DOA): 161 (09/jun), 169 (17/jun), 177 (25/jun), 185 (3/jul), 193 (11/jul), 201 (19/jul), 209 (27/jul), sendo os meses onde ocorre parte do período seco e com pouca presença de nuvens.

\subsection{Métodos}

O Surface Energy Balance Algorithm for Land (SEBAL) (Bastiaanssen et al., 1998) utiliza radiâncias espectrais registradas pelos sensores dos satélites meteorológicos e medidas para calcular o Rn à superfície (Gao et al., 2008). Para o cálculo do Rn através do algoritmo SEBAL, primeiramente foi necessário o cômputo de alguns parâmetros dos produtos de satélites, conforme descrição a seguir:

\section{a) Albedo da superfície $\left(\alpha_{L}\right)$}

O albedo é definido pela razão entre a radiação solar global refletida por cada pixel e a radiação solar incidente no mesmo, em toda a faixa da radiação de onda curta $(0,3$ a 3,0 $\mu \mathrm{m})$ e foi determinado através do método proposto por Liang (2000):

$$
\begin{aligned}
\alpha_{L} & =0,160 \rho_{1}+0,291 \rho_{2}+0,243 \rho_{3}+0,116 \rho_{4} \\
& +0,112 \rho_{5}+0,081 \rho_{7}-0,0015
\end{aligned}
$$

onde $\rho_{1}, \rho_{2}, \ldots, \rho_{7}$ representam as refletâncias monocromáticas relativas a cada uma das sete bandas espectrais do MODIS, distribuídas por meio do produto MODIS/Aqua.

\section{b) Radiação de onda curta incidente $\left(\mathbf{R}_{\mathbf{S} \downarrow}\right)$}

A radiação de onda curta incidente $\left(\mathrm{R}_{\mathrm{S} \downarrow}\right)$ corresponde ao fluxo de radiação solar (direta e difusa) que atinge a superfície terrestre e que para condição de céu claro foi obtida, segundo Allen et al. (2007), a partir da seguinte equação:

$$
R_{S \downarrow}=\frac{S_{0} \cdot \cos \theta_{z} \cdot \tau_{s w}}{d^{2}}
$$


onde $S_{0}$ é a constante solar $\left(1367 \mathrm{~W} \mathrm{~m}^{-2}\right) ; \theta_{\mathrm{z}}$ é ângulo zenital solar, em graus, obtido do produto MODIS/Aqua; $\mathrm{d}^{2}=$ quadrado da distância relativa Terra-Sol, obtido segundo Duffie e Beckman (1991) pela equação:

$$
d^{2}=\frac{1}{1+0,033 \cos (D O A \cdot 2 \pi / 365)}
$$

onde DOA é o dia de ordem do ano. Devido limitações de dados aerológicos sobre a área de estudo, a transmissividade atmosférica $\left(\tau_{\mathrm{sw}}\right)$ foi estimada de acordo com Allen et al. (2002), que assume condições de céu-claro e atmosfera relativamente seca, da seguinte forma:

$$
\tau_{s w}=0,75+2 \cdot 10^{-5} z
$$

onde $\mathrm{z}$ representa a altitude de cada pixel da imagem, obtida através do Modelo de Elevação Digital (DEM) da região de estudo.

\section{c) Radiação de onda longa incidente $\left(\mathbf{R}_{\mathrm{L} \downarrow}\right)$}

A radiação de onda longa incidente, emitida pela atmosfera na direção da superfície $\left(\mathrm{em} \mathrm{W} \mathrm{m}^{-2}\right.$ ), foi obtida através da equação de Stefan-Boltzmann para corpos cinza:

$$
R_{L \downarrow}=\varepsilon_{a} \cdot \sigma \cdot T_{a}^{4}
$$

onde $\mathrm{T}_{\mathrm{a}}$ é a temperatura do ar próximo à superfície, $\sigma$ é a constante de Stefan-Boltzmann $\left(\sigma=5,67 \times 10^{-8} \mathrm{~W} \mathrm{~m}^{-2} \mathrm{~K}^{-4}\right)$ e $\varepsilon_{\mathrm{a}}$ é a emissividade atmosférica, calculada segundo modelo proposto por Bastiaanssen et al. (1998), qual seja:

$$
\varepsilon_{a}=a\left(-\ln \tau_{s w}\right)^{b}
$$

onde $a$ e $b$ são coeficientes de calibração cujos valores, de acordo com Bastiaanssen et al. (1998), são respectivamente iguais a 1,08 e 0,265 .

\section{d) Radiação de onda longa emitida $\left(\mathbf{R}_{\mathrm{L} \uparrow}\right)$}

A radiação de onda longa emitida por cada pixel (em $\mathrm{W} \mathrm{m}^{-2}$ ) foi calculada também segundo a equação de StefanBoltzmann para corpos cinza:

$$
R_{L \uparrow}=\varepsilon_{0} \cdot \sigma \cdot T_{s}^{4}
$$

onde $\varepsilon_{0}$ é a emissividade de cada pixel e $\mathrm{T}_{\mathrm{s}}(\mathrm{K})$ é a temperatura da superfície, as quais foram obtidas a partir do produto MODIS/AQUA. A emissividade da superfície foi considerada igual à média aritmética das emissividades das bandas 31 e 32, conforme proposto por Bisht et al. (2005).

$\mathrm{O}$ albedo utilizado para computar o Rn foi o albedo Liang (2000). Andrade (2008) também utilizou a Equação 1 para o cômputo do albedo que posteriormente foi utilizado para estimativa do Rn segundo o método SEBAL.

\section{e) Saldo de radiação à superfície $\left(\mathbf{R}_{n}\right)$}

O saldo de radiação foi estimado com base na equação a seguir:

$$
R_{n}=R_{S \downarrow}(1-\alpha)+R_{L \downarrow}-R_{L \uparrow}-\left(1-\varepsilon_{0}\right) R_{L \downarrow}
$$

em que $\mathrm{R}_{\mathrm{S} \downarrow}$ é a radiação de onda curta incidente, $\alpha$ é o albedo corrigido de cada pixel, $\mathrm{R}_{\mathrm{L} \downarrow}$ é a radiação de onda longa emitida pela atmosfera na direção de cada pixel, $\mathrm{R}_{\mathrm{L} \uparrow}$ é a radiação de onda longa emitida por cada pixel e $\varepsilon_{0}$ é a emissividade de cada pixel. As densidades de fluxos são expressas em $\mathrm{W} \mathrm{m}^{-2}$. A Figura 2

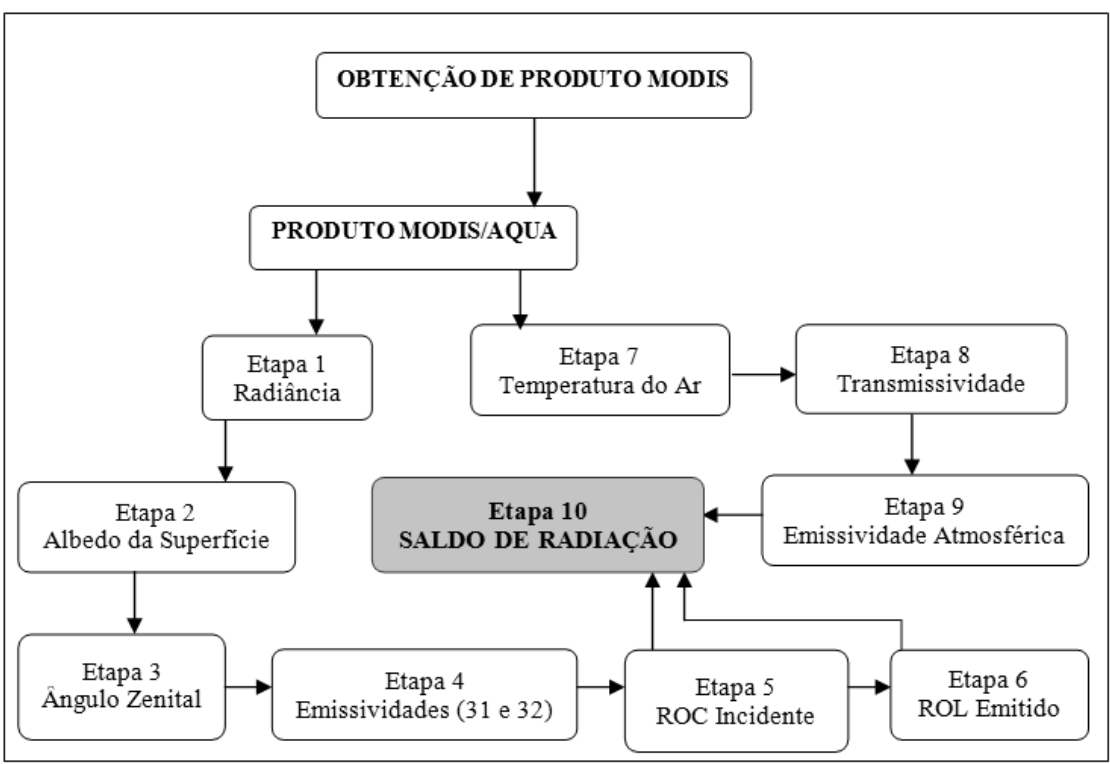

Figura 2 - Fluxograma das etapas para estimar o Rn através do algoritmo SEBAL, utilizando produtos do satélite MODIS/Aqua. 
representa o fluxograma das etapas para estimar o Rn através do algoritmo SEBAL, utilizando produtos do satélite MODIS/Aqua.

\section{f) NDVI (Normalized Difference Vegetation Index)}

O NDVI é um índice que permite identificar a presença de vegetação verde na superfície e caracterizar sua distribuição espacial, como também identificar sua evolução no decorrer do tempo. Embora não seja preciso o cômputo do NDVI para obter o Rn para os produtos MODIS/Aqua, ele foi calculado para comparativos e análises posteriores através da Equação 9:

$$
N D V I=\frac{\left(\rho_{I I}-\rho_{I}\right)}{\left(\rho_{I I}+\rho_{I}\right)}
$$

onde $\rho_{\mathrm{I}}$ e $\rho_{\mathrm{II}}$ correspondem respectivamente às reflectâncias monocromáticas das bandas 01 e 02 do produto MYD09A1 do MODIS/AQUA.

\section{RESULTADOS E DISCUSSÃO}

Com a temperatura da superfície calculada para os dias selecionados foram obtidas as distribuições espaço-temporal da temperatura de superfície, como mostra a Figura 3, delas foram extraídos os valores das coordenadas referentes às duas áreas, que podem ser vistos na Tabela 3. O aumento da temperatura para os dois pontos é bastante notório e esperado, sabendo que as datas de coleta dos dados são de um período seco. No entanto, é importante que se perceba o aumento da temperatura da superfície em números, tendo para a área de floresta um aumento médio de aproximadamente $1{ }^{\circ} \mathrm{C}$ por data para o período e uma diferença superior a $7{ }^{\circ} \mathrm{C}$ entre os DOA 161 (09/jun) e 209 (27/ jul). Para a área de pastagem, onde há uma exposição maior do solo à radiação solar, esse aumento foi menor que o da região de floresta, apresentando uma diferença de aproximadamente $6{ }^{\circ} \mathrm{C}$ do primeiro dia em relação ao último, porém em todas as situações apresentou valores de temperatura maiores que a área de floresta. A média de temperatura na floresta (Rebio) foi de $30,1^{\circ} \mathrm{C}$ com desvio-padrão de $2,6^{\circ} \mathrm{C}$, enquanto que na pastagem (FNS), a temperatura média foi de $32,6^{\circ} \mathrm{C}$ com desvio-padrão de $2,9^{\circ} \mathrm{C}$. Diferenças similares foram encontradas por Aguiar (2007) analisando a temperatura do ar sobre a região estudada. Este resultado indica que existe uma maior absorção de calor na área de pastagem, resultando numa maior taxa de aquecimento do ar sobre a região. Além disso, indicam que ocorre uma alteração no microclima local quando a floresta e substituída por pastagem, levando a um maior aquecimento da atmosfera,
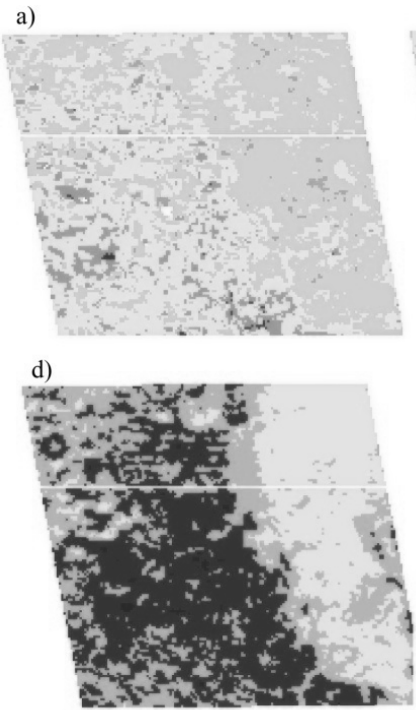

b)

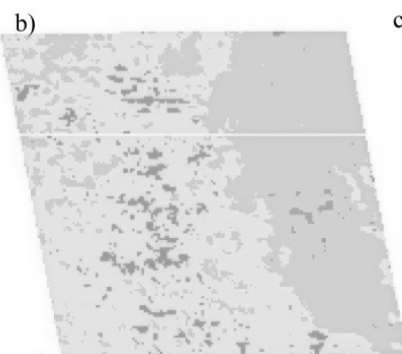

e)
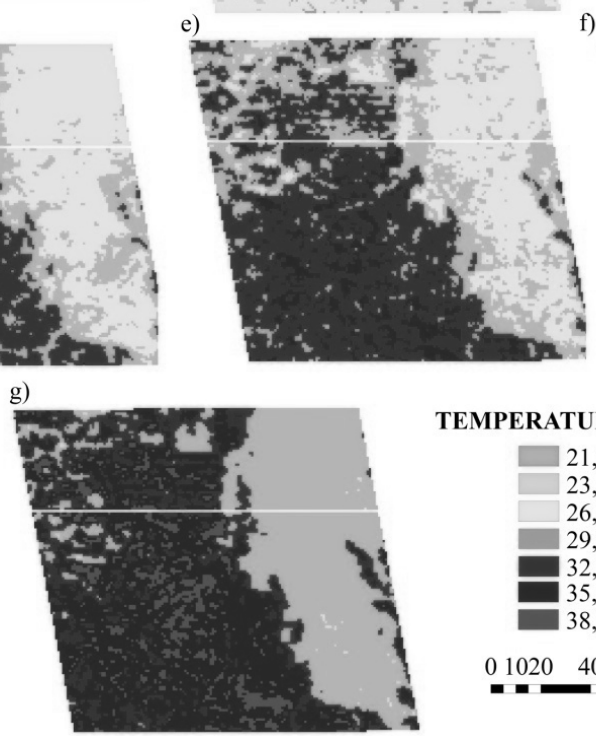

c)

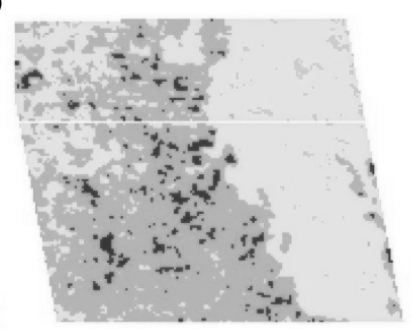

f)

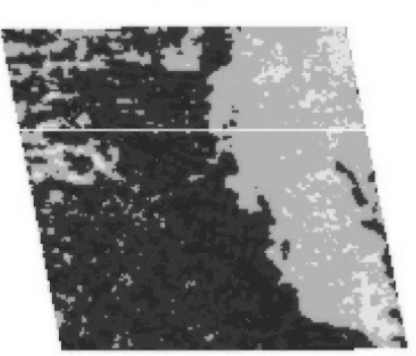

TEMPERATURA DA SUPERFÍCIE $\left({ }^{\circ} \mathrm{C}\right)$

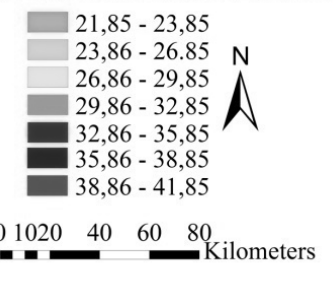

Figura 3 - Mapas da distribuição espaço-temporal da temperatura da superfície na escala Celsius, onde: a) DOA 161; b) DOA 169; c) DOA 177; d) DOA 185; e) DOA 193; f) DOA 201; g) DOA 209. 
Tabela 3 - Temperatura da superfície para os pontos das torres medidos através de produtos MODIS/Aqua

\begin{tabular}{cccc}
\hline DOA & $\begin{array}{c}\text { REBIO } \\
\left({ }^{\circ} \mathbf{C}\right)\end{array}$ & $\begin{array}{c}\text { FNS } \\
\left({ }^{\mathbf{O}} \mathbf{C}\right)\end{array}$ & $\begin{array}{c}\text { DIFERENÇA } \\
\left({ }^{\mathbf{}} \mathbf{C}\right)\end{array}$ \\
\hline 161 & 26,4 & 29,3 & 2,9 \\
169 & 27,6 & 29,4 & 1,8 \\
177 & 28,7 & 30,4 & 1,7 \\
185 & 31,3 & 32,6 & 1,3 \\
193 & 31,0 & 35,3 & 4,3 \\
201 & 32,0 & 35,8 & 3,8 \\
209 & 33,8 & 35,2 & 1,9 \\
Média \pm Desvio & $30,1 \pm 2,6$ & $32,6 \pm 2,9$ & $2,5 \pm 1,2$ \\
Padrão & & &
\end{tabular}
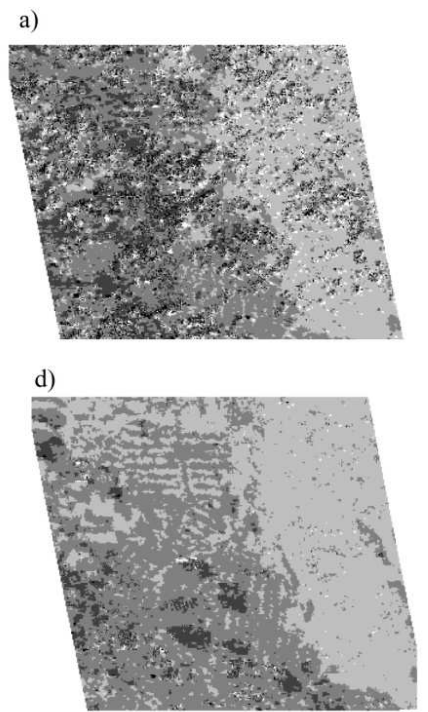

b)

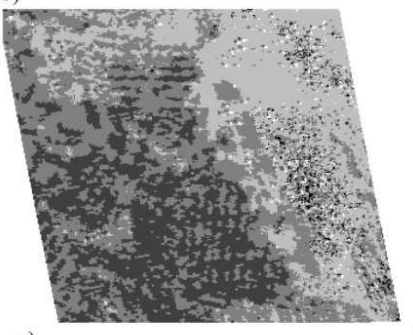

e)

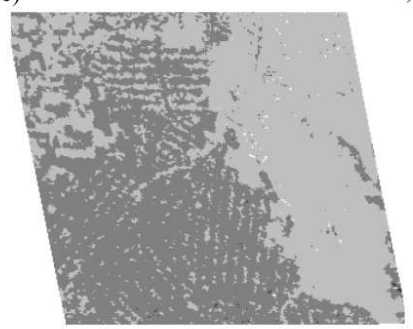

c)

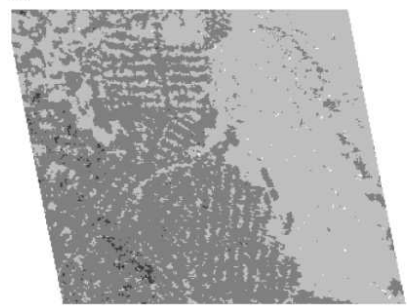

f)

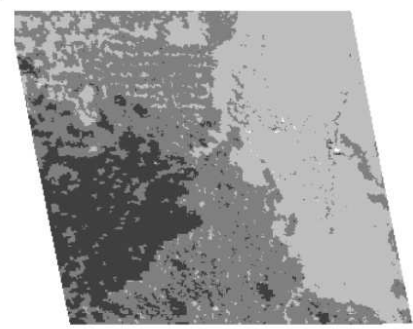

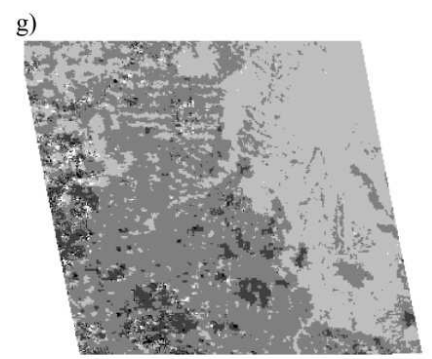

\section{Albedo da Superfície}

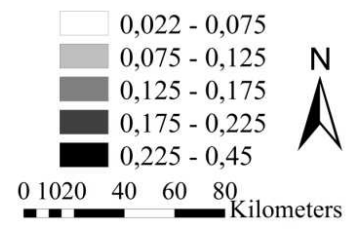

Figura 4 - Mapas do comportamento da distribuição espaço-temporal do albedo da superfície, onde: a) DOA 161; b) DOA 169; c) DOA 177; d) DOA 185; e) DOA 193; f) DOA 201; g) DOA 209.

devido à maior disponibilidade de energia na forma de calor sensível em comparação com a floresta.

Os dados quantitativos do albedo da superfície apresentaram valores de 0,02 a 0,45 , conforme apresentado na Figura 4. Evidencia-se que o valor inferior $(0,02)$ corresponde a erros ou a sombra de nuvens, assim como, o superior $(0,45)$ é produzido por nuvens. Logo, consideram-se esses valores como sendo anômalos ao comportamento do albedo da região estudada. Ainda de acordo com a Figura 4, constata-se que o albedo da superfície da área de pastagem foi sempre maior do que na área de floresta. Esse fato pode ser explicado por dois fatores: o tom da coloração da vegetação e a estrutura vertical característica de cada área. A área de floresta tem vegetação mais escura e mais alta (em média 30 metros), o que acarreta na 
Tabela 4 - Análise comparativa dos valores do albedo para os pontos de localização das torres e o Erro Percentual (EP)

\begin{tabular}{ccccccc}
\hline DOA & $\begin{array}{c}\text { Rebio } \\
\text { (MODIS) }\end{array}$ & $\begin{array}{c}\text { Rebio } \\
\text { (Torre) }\end{array}$ & $\begin{array}{c}\text { EP } \\
\mathbf{( \% )}\end{array}$ & $\begin{array}{c}\text { FNS } \\
\text { (MODIS) }\end{array}$ & $\begin{array}{c}\text { FNS } \\
\text { (Torre) }\end{array}$ & $\begin{array}{c}\text { EP } \\
\text { (\%) }\end{array}$ \\
\hline 161 & 0,104 & 0,117 & -11 & 0,189 & 0,196 & -6 \\
169 & 0,104 & 0,120 & -13 & 0,189 & 0,193 & -2 \\
177 & 0,096 & 0,124 & -22 & 0,159 & 0,196 & -19 \\
185 & 0,110 & 0,122 & -10 & 0,158 & 0,199 & -21 \\
193 & 0,109 & 0,123 & -11 & 0,175 & 0,200 & -12 \\
201 & 0,113 & 0,121 & -7 & 0,188 & 0,204 & -8 \\
209 & 0,124 & 0,115 & 8 & 0,182 & 0,205 & -11 \\
$\begin{array}{c}\text { Média } \pm \text { Desvio } \\
\text { Padrão }\end{array}$ & $0,109 \pm 0,009$ & $0,120 \pm 0,003$ & - & $0,177 \pm 0,014$ & $0,199 \pm 0,004$ & - \\
\hline
\end{tabular}

menor refletividade de energia, diferente da área de pastagem, por ser caracterizada por solo exposto, vegetação gramínea e arbustiva, detém menor absorção de energia solar que chega a ser absorvida.

Em comparação aos dados medidos na área de estudo pelas torres micrometeorológicas, em todos os casos foram registrados valores superiores para as duas áreas, exceto para a última data dos dados da Rebio. Na Tabela 4 estão descritos os valores do albedo medidos pelas torres e estimados através das imagens do MODIS/Aqua, para os dois pontos estudados. Para a região de floresta, o valor médio medido do albedo foi da ordem de $12 \%$ e o estimado de $11 \%$. A Tabela 4 indica pouca diferença entre a estimativa, sugerindo que situações de nebulosidade escassa podem continuar próximas daquelas de céu claro. Esses resultados são compatíveis com os valores obtidos em alguns trabalhos anteriormente realizados sobre áreas de florestas da Região Amazônica, que resultaram nos seguintes valores médios: 13,4\% (Culf et al.,1995), 12,2\% (Shuttleworth et al., 1984), 13,1\% (Bastable et al., 1993). De acordo com Rotenberg e Yaki (2010), o valor médio do albedo de florestas tropicais, temperadas e semiáridas é da ordem de $10 \%$.

Verifica-se que o Erro Percentual (EP), entre os valores observados e estimados a partir de dados MODIS, mostrou valores máximos e mínimos de $7 \%$ e $22 \%$, respectivamente, sendo o menor EP para o DOA 201 e o maior para o DOA 177. Os valores médios do albedo medido para a pastagem foram da ordem de $20 \%$ e o estimado de $18 \%$. Esses resultados concordam com Aguiar (2007) que encontrou albedo médio anual na área de pastagem de $21 \%$, quase o dobro do observado em área de floresta que foi de $11,7 \%$. Verifica-se ainda que esses resultados são compatíveis com valores encontrados em outros trabalhos realizados na mesma região, cujos valores médios são os seguintes: 16,3\% (Bastable et al., 1993), 19\% (Fisch et al., 1994), 18\% (Culf et al., 1995). Na comparação entre os valore observados e estimados a partir dos dados MODIS verifica-se que os valores máximos e mínimos do EP obtidos para a região de pastagem foram $21 \%$ e $2 \%$, respectivamente para os DOA 185 e 169. Analisando a Tabela 4, é possível observar que os valores (estimados e medidos) do albedo de superfície têm pouca variabilidade temporal, o que pode ser observado através de seus valores médios e desvio-padrão. Os valores apresentados indicam coerência aceitável entre ambas as metodologias de obtenção do albedo da região estudada.

Mesmo havendo diferenças entre os valores de albedo encontrados tanto na floresta como na pastagem, eles nos fornecem um indicativo quantitativo da redução na radiação solar global absorvida pela superfície quando a cobertura vegetal de floresta é substituída por pastagem ou culturas agrícolas e, o sensoriamento remoto apresentou-se como uma ferramenta útil na obtenção da distribuição espacial dessa variável ambiental.

$\mathrm{Na}$ Tabela 5, podem ser observados os valores de NDVI para a localização das torres micrometeorológicas. Esses valores mostram que para a área de floresta há uma pequena diminuição nesse índice, indicando para o primeiro dia da amostra o valor de 0,89 e mínimo de 0,83 para o último DOA analisado, ou seja, uma diferença de 0,05 . Na área de pastagem, a variação do NDVI foi mais intensa, alcançando uma diferença de aproximadamente 0,30 , e tendo valores máximos e mínimos de

Tabela 5: Valores de NDVI para os pontos das torres

\begin{tabular}{ccc}
\hline DOA & REBIO (MODIS) & FNS (MODIS) \\
\hline 161 & 0,89 & 0,74 \\
169 & 0,88 & 0,70 \\
177 & 0,87 & 0,65 \\
185 & 0,87 & 0,65 \\
193 & 0,88 & 0,57 \\
201 & 0,87 & 0,47 \\
209 & 0,83 & 0,44 \\
Média \pm Desvio & $0,87 \pm 0,02$ & $0,60 \pm 0,11$ \\
Padrão & & \\
\hline
\end{tabular}


0,74 e 0,44 para os DOA 161 e DOA 209, respectivamente. Esta diminuição ocorre devido à intensificação da seca que afeta o desenvolvimento das gramíneas (pastagens), no entanto, o valor mínimo de 0,44 , pode também estar relacionado às atividades de queimadas na área, que reduzem, consideravelmente, os valores de NDVI. Assim, a área de pastagem apresentou menores valores de NDVI, devido ao déficit hídrico, enquanto a área de floresta apresentou valores mais elevados de NDVI, porque nessas regiões podem ser detectados maiores conteúdos de água do solo e da biomassa verde. O comportamento espaço-temporal do NDVI está apresentado na Figura 5.

As Tabelas 6, 7 e 8 mostram a comparação, além dos valores médios e desvio-padrão, entre os valores medidos e os valores estimados, assim como, o EP para as componentes radiativas do saldo de radiação à superfície. Na Tabela 6 , que apresenta os valores da $\mathrm{R}_{\mathrm{L} \downarrow}$ (radiação de onda longa emitida a)

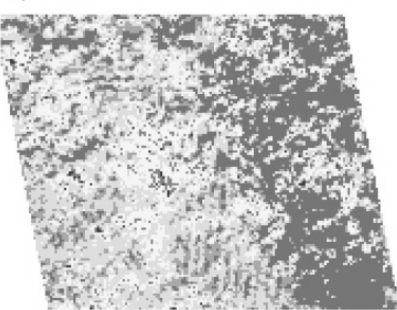

d)

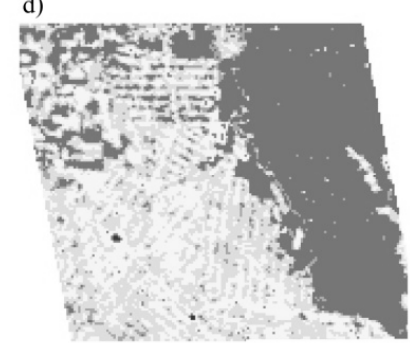

b)

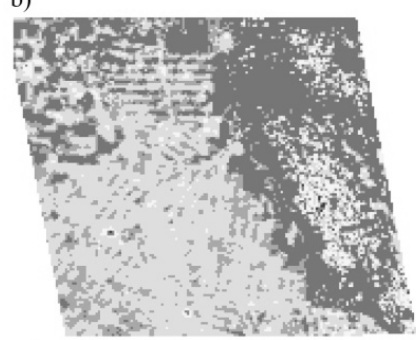

e)

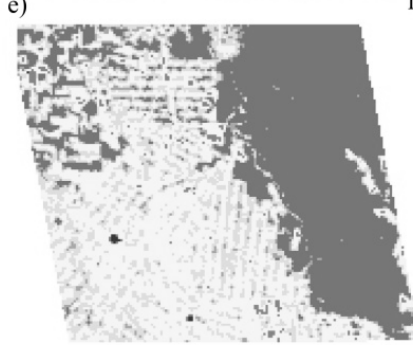

c)

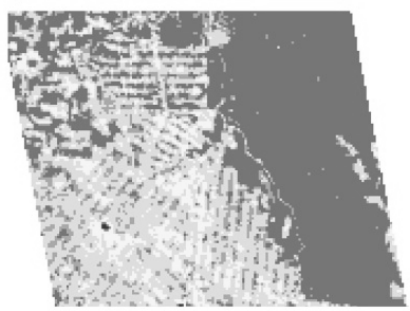

f)

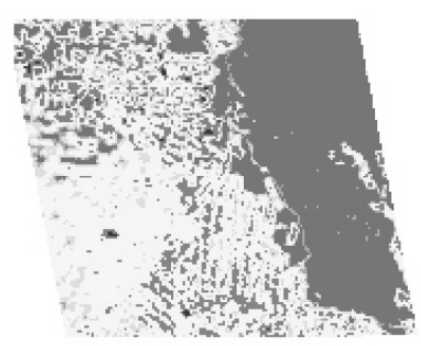

g)

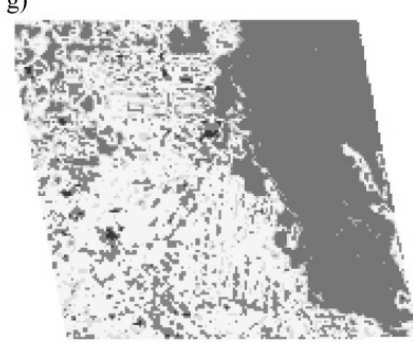

NDVI

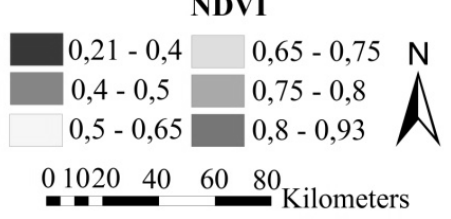

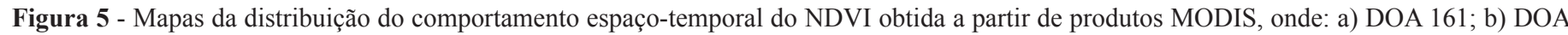
169; c) DOA 177; d) DOA 185; e) DOA 193; f) DOA 201; g) DOA 209.

Tabela 6 - Análise comparativa dos valores da $\mathrm{R}_{\mathrm{L} \downarrow}\left(\mathrm{W} \mathrm{m}^{-2}\right)$ obtidos através do SEBAL e das torres micrometeorológicas, bem como o Erro Percentual (EP)

\begin{tabular}{ccccccc}
\hline DOA & $\begin{array}{c}\text { Rebio-MODIS } \\
\left(\mathbf{W} \mathbf{~ m}^{-\mathbf{2}}\right)\end{array}$ & $\begin{array}{c}\text { Rebio-Torre } \\
\left(\mathbf{W} \mathbf{~ m}^{-\mathbf{2}}\right)\end{array}$ & $\begin{array}{c}\text { EP } \\
(\mathbf{\%})\end{array}$ & $\begin{array}{c}\text { FNS-MODIS } \\
\left(\mathbf{W} \mathbf{~ m}^{-\mathbf{2}}\right)\end{array}$ & $\begin{array}{c}\text { FNS-Torre } \\
\left(\mathbf{W} \mathbf{~ m}^{-\mathbf{2}}\right)\end{array}$ & $\begin{array}{c}\text { EP } \\
(\mathbf{\%})\end{array}$ \\
\hline 161 & 345,74 & 383,33 & -10 & 361,77 & 431,00 & -16 \\
169 & 375,45 & 355,94 & 5 & 377,38 & 402,22 & -6 \\
177 & 358,40 & 340,73 & 5 & 359,23 & 377,44 & -5 \\
185 & 372,43 & 378,75 & -2 & 377,16 & 414,04 & -9 \\
193 & 365,05 & 351,56 & 4 & 368,95 & 396,30 & -7 \\
201 & 345,8 & 375,02 & -8 & 366,55 & 422,16 & -13 \\
209 & 368,83 & 392,45 & -6 & 367,21 & 435,55 & -16 \\
Média \pm Desvio & & & & & & \\
Padrão & $361,7 \pm 12,1$ & $368,2 \pm 18,9$ & - & $368,3 \pm 7,0$ & $411,2 \pm 20,6$ & - \\
\hline
\end{tabular}


Tabela 7 - Análise comparativa dos valores da $\mathrm{R}_{\mathrm{L} \uparrow}\left(\mathrm{W} \mathrm{m}^{-2}\right)$ obtidos através do SEBAL e das torres micrometeorológicas, bem como o Erro Percentual (EP).

\begin{tabular}{|c|c|c|c|c|c|c|}
\hline DOA & $\begin{array}{l}\text { Rebio-MODIS } \\
\qquad\left(\mathrm{W} \mathrm{m}^{-2}\right)\end{array}$ & $\begin{array}{l}\text { Rebio-Torre } \\
\qquad\left(\mathbf{W} \mathbf{~ m}^{-2}\right)\end{array}$ & $\begin{array}{c}\text { EP } \\
(\%)\end{array}$ & $\begin{array}{c}\text { FNS-MODIS } \\
\left(\mathbf{W} \mathbf{~ m}^{-2}\right)\end{array}$ & $\begin{array}{c}\text { FNS-Torre } \\
\left(\mathbf{W} \mathbf{m}^{-2}\right)\end{array}$ & $\begin{array}{l}\text { EP } \\
(\%)\end{array}$ \\
\hline 161 & 446,70 & 481,95 & -7 & 466,70 & 518,25 & -10 \\
\hline 169 & 453,65 & 458,27 & -1 & 467,55 & 495,70 & -6 \\
\hline 177 & 465,50 & 449,13 & 3 & 473,76 & 477,73 & 1 \\
\hline 185 & 481,20 & 477,07 & 1 & 487,64 & 512,43 & -5 \\
\hline 193 & 474,20 & 483,14 & -2 & 505,50 & 521,07 & -3 \\
\hline 201 & 446,70 & 492,44 & -9 & 508,51 & 533,84 & -4 \\
\hline 209 & 499,12 & 491,36 & 2 & 504,70 & 557,18 & -9 \\
\hline \multicolumn{7}{|c|}{ Média \pm Desvio } \\
\hline Padrão & $466,7 \pm 19,5$ & $476,2 \pm 16,5$ & - & $487,8 \pm 18,6$ & $516,6 \pm 25,6$ & - \\
\hline
\end{tabular}

Tabela 8 - Análise comparativa dos valores da $\left(\mathrm{R}_{\mathrm{S} \downarrow}\right)\left(\mathrm{W} \mathrm{m}^{-2}\right)$ obtidos através do SEBAL e através das torres micrometeorológicas, bem como o Erro Percentual (EP).

\begin{tabular}{|c|c|c|c|c|c|c|}
\hline DOA & $\begin{array}{c}\text { Rebio -MODIS } \\
\left(\mathrm{W} \mathrm{m} \mathrm{m}^{-2}\right)\end{array}$ & 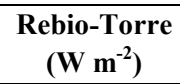 & $\begin{array}{c}\text { EP } \\
(\%)\end{array}$ & $\begin{array}{c}\text { FNS -MODIS } \\
\left(\mathrm{W} \mathrm{m}^{-2}\right)\end{array}$ & $\begin{array}{c}\text { FNS-Torre } \\
\left(\mathbf{W} \mathbf{m}^{-2}\right)\end{array}$ & $\begin{array}{l}\text { EP } \\
(\%)\end{array}$ \\
\hline 161 & 800,82 & 810,00 & -1 & 800,31 & 855,00 & -6 \\
\hline 169 & 707,06 & 701,00 & 1 & 706,00 & 687,80 & 3 \\
\hline 177 & 802,19 & 820,00 & -2 & 801,71 & 803,00 & $-0,2$ \\
\hline 185 & 718,11 & 545,50 & 31 & 717,64 & 716,00 & 0,2 \\
\hline 193 & 819,32 & 842,00 & -3 & 818,70 & 818,00 & 0,1 \\
\hline 201 & 741,37 & 744,00 & $-0,4$ & 741,65 & 733,00 & 1 \\
\hline 209 & 846,48 & 622,70 & 36 & 846,71 & 906,00 & -6 \\
\hline \multicolumn{7}{|c|}{ Média \pm Desvio } \\
\hline Padrão & $776,5 \pm 53,9$ & $726,5 \pm 110,7$ & - & $776,1 \pm 54,1$ & $788,4 \pm 79,4$ & - \\
\hline
\end{tabular}

pela atmosfera), pode ser observado, a partir do EP, que houve uma subestimativa dos dados estimados em relação aos dados medidos para a área de pastagem, que variou entre 5\% e 16\%, ou seja, os valores estimados pelo SEBAL foram inferiores aos medidos pelas torres nessa área. No caso da área de floresta houve valores superestimados $(4 \%$ e $5 \%)$ e subestimados $(2 \%$ a $10 \%$ ).

A Tabela 7 mostra que o comportamento da radiação de onda longa emitida pela superfície $\left(\mathrm{R}_{\mathrm{L} \uparrow}\right)$ estimada pelo SEBAL também foi satisfatório ao ser comparado aos dados medidos pelas torres para as duas áreas, utilizando um piranômetro modelo CG1 (Kipp \& Zonen), apresentando valores de EP máximos e mínimos de $9 \%$ e 1\% para a Rebio (floresta) e de $10 \%$ e $1 \%$ para a FNS (pastagem).

A Tabela 8 apresenta a comparação entre os dados estimados e medidos (SEBAL e torre, respectivamente) de radiação de onda curta incidente $\left(R_{S \downarrow}\right)$, onde podem ser observados os menores valores de EP, ou seja, onde houve uma maior aproximação entres os valores, porém, os DOA 185 e 209 da área de floresta apresentaram um EP de 31\% e $36 \%$, respectivamente. Este fato pode estar associado a algum tipo de manutenção necessária (ou recém-aplicada) nos sensores que pode ter ocorrido nos dias analisados ou até mesmo por alguma interferência natural, como sujeira na lente do instrumento como mancha por queda de algum fruto das árvores, entre outros fatores naturais. Dos resultados apresentados nessa tabela, pode ser evidenciado que, mesmo o Estado de Rondônia apresentar elevado teor de vapor de água na atmosfera, a aplicação da equação simplificada para obtenção da transmissividade atmosférica sobre a região estudada não impactou, consideravelmente, os resultados obtidos através do MODIS/Aqua, visto que foram coerentes com os valores medidos à superfície.

Todas essas observações relacionadas às radiações refletem diretamente nos resultados do saldo de radiação. Os valores de Rn obtidos a partir do algoritmo SEBAL, aplicado para região de clima tropical úmido apresentou valores bem próximos aos medidos pelas torres micrometeorológicas que estão implantadas no local. Na Tabela 9 estão comparados o comportamento temporal pontual do $\mathrm{Rn}$ computado pelo SEBAL e o obtido pelas torres micrometeorológicas, como também o EP. A partir desses resultados pode ser observado que a aplicabilidade de produtos orbitais para a obtenção desse tipo de produto é muito confiável e funcional, pois, como podem 
ser observados, os EP são baixos, ou seja, variando entre 0 e 10\%, com exceção dos DOA 185 e 209 para a área de floresta, porém constata-se que o valor fora do padrão não é proveniente do SEBAL, mas sim, dos dados da torre, um erro acumulativo decorrente da radiação de onda curta incidente, conforme anteriormente observado.

A Figura 6 mostra o comportamento espaço-temporal do $\mathrm{Rn}$. Nota-se nas referidas figuras que as diferenças entre o Rn das áreas de pastagem e o Rn das áreas de floresta variam de 50 a 100 $\mathrm{W} \mathrm{m}^{-2}$. Esses resultados expõem o impacto da substituição da floresta por áreas de atividades agropecuárias. Além do impacto radiativo, também deve ser levado em consideração o impacto quanto às emissões de gases de efeito estudo. De acordo com Houghton et al. (2001) a expansão agrícola é responsável por um incremento significativo da transferência de $\mathrm{CO}_{2}, \mathrm{~N}_{2} \mathrm{O}$ e $\mathrm{CH}_{4}$ da biosfera para a atmosfera. Estima-se que, globalmente,

Tabela 9 - Análise comparativa dos valores do $\mathrm{Rn}\left(\mathrm{W} \mathrm{m}^{-2}\right)$ obtidos através do SEBAL e através das torres micrometeorológicas, bem como o Erro Percentual (EP),

\begin{tabular}{|c|c|c|c|c|c|c|}
\hline DOA & 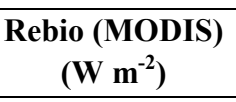 & 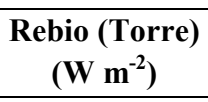 & $\begin{array}{l}\text { EP } \\
(\%)\end{array}$ & $\begin{array}{c}\text { FNS (MODIS) } \\
\left(\mathbf{W ~ m}^{-2}\right)\end{array}$ & 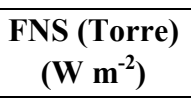 & EP (\%) \\
\hline 161 & 611,20 & 616,16 & 1,0 & 538,53 & 599,44 & $-10,0$ \\
\hline 169 & 529,80 & 514,10 & 3,0 & 480,23 & 461,81 & 4,0 \\
\hline 177 & 609,80 & 609,70 & 0,0 & 556,44 & 545,21 & 2,0 \\
\hline 185 & 528,20 & 380,83 & 38,0 & 489,85 & 474,61 & 3,0 \\
\hline 193 & 611,72 & 606,92 & 1,0 & 547,31 & 529,43 & 3,0 \\
\hline 201 & 537,57 & 536,50 & 0,2 & 475,10 & 471,72 & 0,7 \\
\hline 209 & 615,27 & 452,10 & 36,0 & 549,22 & 598,18 & $-8,0$ \\
\hline \multicolumn{7}{|c|}{ Média \pm Desvio } \\
\hline Padrão & $577,6 \pm 43,0$ & $530,9 \pm 89,7$ & - & $519,5 \pm 36,0$ & $525,8 \pm 58,7$ & - \\
\hline
\end{tabular}
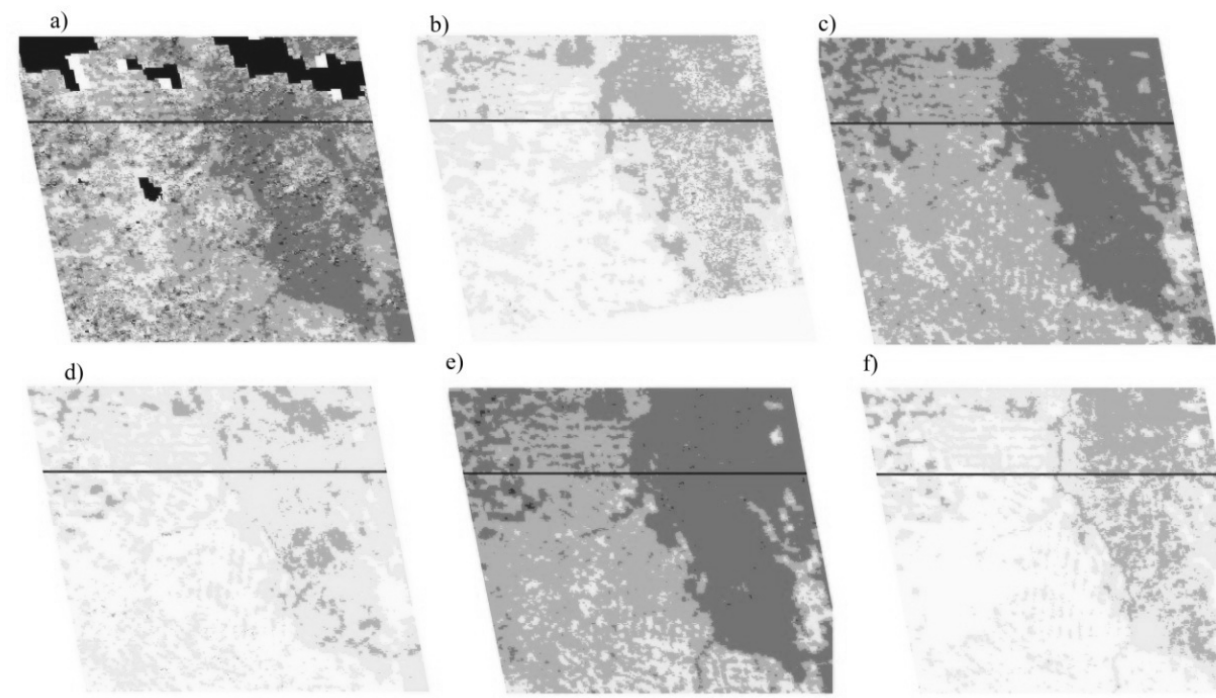

e) f)
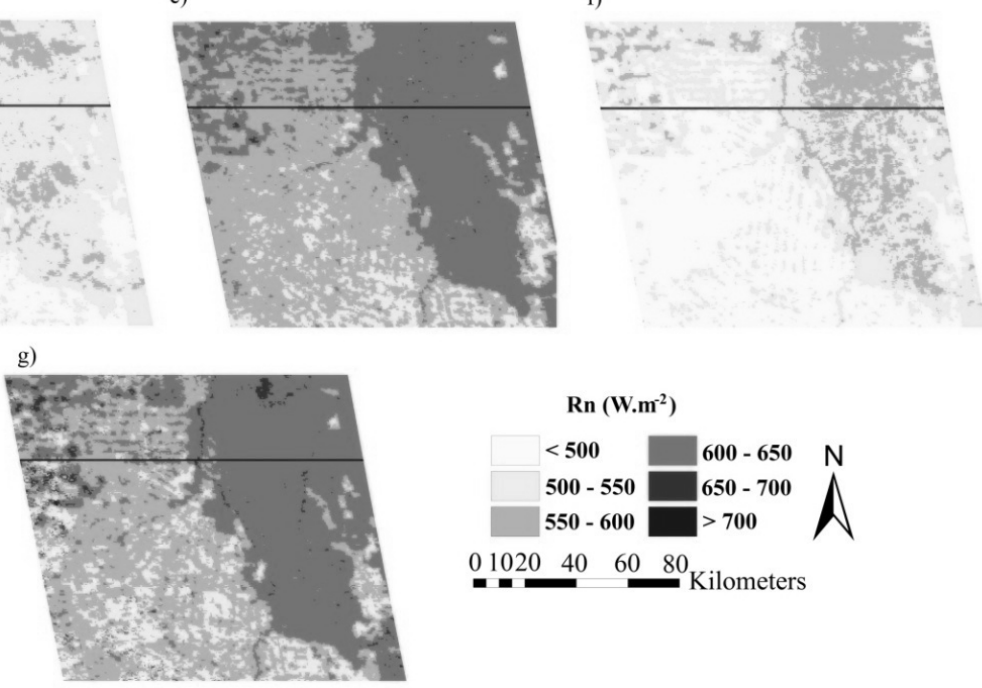

Figura 6 - Mapas da distribuição do comportamento espaço-temporal do saldo de radiação à superfície, obtido a partir de produtos MODIS, onde: a) DOA 161; b) DOA 169; c) DOA 177; d) DOA 185; e) DOA 193; f) DOA 201; g) DOA 209. 
a agricultura contribui por $21-25 \%$ do total das emissões de $\mathrm{CO}_{2} ; 55-60 \%$ de $\mathrm{CH}_{4}$ e $65-80$ de $\mathrm{N}_{2} \mathrm{O}$.

As aplicações de técnicas de sensoriamento remoto orbital para climas tropicais úmidos são difíceis. O principal problema é a constante presença de nuvens devido ao processo convectivo que é um importante mecanismo de aquecimento da atmosfera tropical (Santos et al., 2011). Sabe-se que dados medidos são fundamentais na validação de resultados de modelos meteorológicos, devido serem mais realísticos para um local específico. Entretanto, eles apresentam dificuldades operacionais, sua obtenção requer um alto investimento financeiro e, usualmente, limitam-se às pequenas áreas e não tem representatividade espacial. Dessa forma, o sensoriamento remoto se apresenta como um ferramenta importante na obtenção das variações temporais e espaciais das características físicas e biológicas da superfície, por exemplo, o Rn, albedo e NDVI, melhorando assim, as validações das parametrizações dos modelos físicos-matemáticos utilizados nos estudos ambientais.

\section{CONCLUSÕES}

Os resultados apresentados nesse estudo sugerem que, na Amazônia, a substituição de floresta por pastagem acarreta em um aumento de, aproximadamente, $2,5^{\circ} \mathrm{C}$ na temperatura da superfície do solo e um considerável aumento do albedo da superfície, de aproximadamente $12 \%$ para $18 \%$. Eltahir e Bras (1994) encontraram resultados similares. Também é evidente um decréscimo no saldo de radiação (Rn) da ordem de 60 $\mathrm{W} \mathrm{m}{ }^{-2}$ (aproximadamente 10\%), corroborando com Gash e Nobre (1997). Esses resultados indicam que o desflorestamento resulta em menor absorção da radiação solar pela superfície e, consequentemente, o Rn é reduzido sobre a região desmatada. Outro importante efeito, que foi discutido por Eltahir e Bras (1994), é a redução do comprimento de rugosidade das áreas desmatadas, afetando assim, o transporte turbulento de calor e vapor de água próximo à superfície. A combinação da redução do Rn e do comprimento de mistura, sobre as áreas desmatadas, resulta numa menor evapotranspiração dessas áreas. A redução na evapotranspiração acarreta numa camada limite mais seca, reduzindo assim, a disponibilidade de energia para convecção e precipitação.

Estes resultados apresentam o sensoriamento remoto orbital como uma ferramenta importante e eficaz para ser usado em estudos ambientais, e para obter coerentes variações temporais e espaciais de características da superfície, ajudando na melhoria e na validação de parametrizações de modelos físico-matemáticos. O bom desempenho das estimativas confirma a eficiência do método em regiões tropicais úmidas, visto que o mesmo foi concebido, calibrado e validado, predominantemente em regiões desérticas, áridas e semiáridas.
No entanto, as aplicações de técnicas de sensoriamento remoto em climas tropicais úmidos ainda são difíceis, porque existe constante presença de nuvens, devido ao processo convectivo. Estudos futuros objetivam a aplicação de metodologias mais robustas que extrapolem a estimativa instantânea de Rn para a obtenção do valor diário.

\section{AGRADECIMENTOS}

Os autores agradecem ao CNPq pela bolsa de Produtividade em Pesquisa cedida ao primeiro autor e ao Programa de Grande Escala da Biosfera-Atmosfera na Amazônia (LBA/INPA) pela concessão dos dados medidos à superfície

\section{REFERÊNCIAS BIBLIOGRÁFICAS}

AGUIAR, L. J. G. Balanço de radiação em áreas de floresta e de pastagem em Rondônia. Dissertação de Mestrado, Viçosa, MG, 2007. $70 \mathrm{p}$.

AHMAD, M. D., TURRAL, H., NAZEER, A. Diagnosing irrigation performance and water productivity through satellite remote sensing and secondary data in a large irrigation system of Pakistan. Agricultural Water Management, v. 96, p. 551-564, 2009.

ALLEN, R. G.; TASUMI, M.; TREZZA, R. Satellite-based energy balance for mapping evapotranspiration with internalized calibration (METRIC) - Model. Journal of Irrigation and Drainage Engineering, v. 133, n. 4, p.380394, 2007.

ALLEN, R.G., TASUMI, M., TREZZA, R., WATERS, R., BASTIAANSSEN, W. Surface Energy Balance Algorithm for Land (SEBAL) - Advanced training and Users Manual. Idaho. 2002.

ANDRADE, R. G. Aplicação do algoritmo SEBAL na estimativa da evapotranspiração e da biomassa acumulada da cana-de-açúcar, 164p. Tese (Doutorado em Meteorologia Agrícola). Universidade Federal de Viçosa MG, 164p, 2008.

BASTABLE, H. G., SHUTTLEWORTH, W. J., DALLAROSA, R. L. G., FISCH, G., NOBRE, C A. Observations of climate albedo, and surface radiation over cleared and undisturbed Amazonian forest. International Journal of Climatology, v.13, p.783-796, 1993.

BASTIAANSSEN, W. G. M. SEBAL-based sensible and latent heat fluxes in the irrigated Gediz Basin, Turkey. Journal of Hydrology, v. 229, p. $87-100,2000$.

BASTIAANSSEN, W. G. M., MENENTI, M., FEDDES, R. A., HOLTSLAG, A. A. M. A remote sensing surface energy balance algorithm for land (SEBAL) 1. Formulation. Journal of Hydrology, v. 212-213, p.198-212. 1998. 
BISHT, G., BRAS, R. L. Estimation of net radiation from the Moderate Resolution Imaging Spectroradiometer over the continental United States. IEEE Transactions on Geoscience and Remote Sensing, v. 49, n. 6, p. 2448 2462, 2011.

BISHT, G., BRAS, R. L. Estimation of net radiation from the MODIS data under all sky conditions: Southern Great Plains case study. Remote Sensing of Environment, v. 114, p. $1522-1534,2010$.

BISHT, G., VENTURINI, V., ISLAM, S., JIANG, L. Estimation of the net radiation using MODIS (Moderate Resolution Imaging Spectroradiometer) data for clear sky days. Remote Sensing of Environment, v. 97, p. 52 - 67, 2005.

CAI, X. L., SHARMA, B. R. Integrating remote sensing, census and weather data for an assessment of rice yield, water consumption and water productivity in the Indo-Gangetic river basin. Agricultural Water Management, v. 97, p. 309-316, 2010.

CULF A. D., FISCH, G., HODNETT, M. H. The albedo of Amazonian forest and ranchland. Journal of Climate, v. 6, p. 1544-1554, 1995.

DUFFIE, J. A.; BECKMAN, W. A. Solar engineering of thermal process, $2^{\text {nd }}$ Ed., Wiley, N. Y. 1991.

ELTAHIR, E. A. B., BRAS, R. L. Sensitivity of regional climate to deforestation in the Amazon basin. Advances in Water Resources, v. 17, p. 101-115, 1994.

FERREIRA, A. S.; MEIRELLES, M. S. P. Implementação preliminar do modelo SEBAL para estimativa da evapotranspiração na Mesorregião do Sul Goiano. Anais XV Simpósio Brasileiro de Sensoriamento Remoto, 2011.

FISCH, G., WRIGHT, I. R., BASTABLE, H. G. Albedo of tropical grass: A case study of pre- and post-burning. International Journal of Climatology, v. 14, p. 102-107, 1994.

GAO, Y., LONG, D., LI, Z. L. Estimation of daily actual evapotranspiration from remotely sensed data under complex terrain over the upper Chao river basin in North China. International Journal of Remote Sensing, v. 29, p. 3295-3315, 2008.

GASH, J. H. C., NOBRE, C. A. Climatic effect of Amazonian deforestation: some results from ABRACOS. Bulletim of American Meteorological Society, v. 78, p. 823-830, 1997.

HOUGHTON, J. T., DING, Y., GRIGGS, D. J., NOGUER, M., VAN DER LINDEN, P. J., DAI, X., MASKELL, K., JOHNSON, C. A. Climate Change 2001: The Scientific Basis. Cambridge University Press, 2001. 83p.

IPCC. Climate Change and Water. Technical Paper of the Intergovernmental Panel on Climate Change, Geneva, Switzerland, 2008.

LIANG, S. Narrowband to broadband conversions of land surface albedo: I. Algorithms. Remote Sensing of Environment. v. 76, p. 213-238, 2000.

MENDONÇA, J. C.; ANDRÉ, R. G. B.; SOUSA, E. F.; SILVA, B. B. Aplicação do algoritmo SEBAL e imagens MODIS para estimativa do fluxo de calor do solo $(G)$ na região Norte Fluminense, RJ, Brasil. Anais XIV Simpósio Brasileiro de Sensoriamento Remoto, 2009.

RIMÓCZI-PAÁL, A. Mapping of radiation balance components for region of Hungary using satellite information. Physics and Chemistry of the Earth, v.30, p. 151-158, 2005.

ROTENBERG, E., YAKIR, D. Contribution of Semi-Arid Forests to the Climate System. Science, v. 327, p. $451-$ 454, 2010.

ROY, S. B., AVISSAR, R. Impact of land use/land cover change on regional hydrometeorology in Amazonia. Journal of Geophysical Research, v. 107, n. D20, 8037, 2002.

RYU, Y., KANG, S., MOON, S., KIM, J. Evaluation of land surface radiation balance derived from moderate resolution imaging spectroradiometer (MODIS) over complex terrain and heterogeneous landscape on clear sky days. Agricultural and Forest Meteorology, v.148, p. 1538-1552, 2008.

SAMANI, Z., BAWAZIR, A. S., BLEIWEISS, M., SKAGGS, R., TRAN, V. D. Estimating Daily Net Radiation over Vegetation Canopy through Remote Sensing and Climatic Data. Journal of Irrigation and Drainage Engineering, v.133, p. 291-297, 2007.

SANTOS, C. A. C., NASCIMENTO, R. L., RAO, T. V. R. Net radiation estimation under pasture and forest in Rondônia, Brazil, with TM Landsat 5 images. Atmósfera, v.24, p.435 - 446, 2011.

SHUTTLEWORTH, W. J. et al. Observations of radiation exchange above and below Amazonian forest. Quarterly Journal of the Royal Meteorological Society, v. 466, p. 1163-1169, 1984.

VON RANDOW, C., MANZI, A. O., KRUIJT, B., OLIVEIRA, P. J., ZANCHI, F. B., SILVA, R. L., HODNETT, M. G., GASH, J. H. C., ELBERS, J. A., WATERLOO, M. J., CARDOSO, F. L., KABAT, P. Comparative measurements and seasonal variations in energy and carbon exchange over forest and pasture in South West Amazonia. Theoretical and Applied Climatology, v. 78, p. 5-26, 2004.

WANG, W., LIANG, S. Estimation of high-spatial resolution clear-sky longwave downward and net radiation over land surfaces from MODIS data. Remote Sensing of Environment, v.113, p. 745-754, 2009.

ZHANG, X, BERHANE, T, SEIELSTAD, G. Comparision of Landsat and MODIS estimates of heat fluxes: effect of surface heterogeneity. Geoscience and Remote Sensing Symposium, 2008. IGARSS 2008. IEEE International. v. 3. p III - 759 - III - 762. 